\title{
Realization of a gravity-resonance-spectroscopy technique
}

\author{
Tobias Jenke ${ }^{1}$, Peter Geltenbort ${ }^{2}$, Hartmut Lemmel ${ }^{1,2}$ and Hartmut Abele ${ }^{1,3,4 \star}$
}

\begin{abstract}
Spectroscopy is a method typically used to assess an unknown quantity of energy by means of a frequency measurement. In many problems, resonance techniques ${ }^{1,2}$ enable high-precision measurements, but the observables have generally been restricted to electromagnetic interactions. Here we report the application of resonance spectroscopy to gravity. In contrast to previous resonance methods, the quantum mechanical transition is driven by an oscillating field that does not directly couple an electromagnetic charge or moment to an electromagnetic field. Instead, we observe transitions between gravitational quantum states when the wave packet of an ultra-cold neutron couples to the modulation of a hard surface as the driving force. The experiments have the potential to test the equivalence principle ${ }^{3}$ and Newton's gravity law at the micrometre scale $e^{4,5}$.
\end{abstract}

Generally, a quantum mechanical system that is described by two states can be understood in analogy to a spin- $1 / 2$ system, where the time development is described by the Bloch equations, assuming two states of a fictitious spin in the multiplet, similar to spin-up and spin-down states. In magnetic resonance of a standard spin-1/2 system, the energy splitting results in the precession of the related magnetic moment in the magnetic field. Transitions between the two states are driven by a transverse magnetic radio frequency field. Similar concepts can be applied to any driven two-level system, for example in optical transitions with light fields. Variations are inherently connected to high-precision measurements such as atomic clocks ${ }^{6}$, atom interferometry ${ }^{7}$, nuclear magnetic resonance ${ }^{8}$, quantum metrology ${ }^{9}$ and the related spin-echo technique ${ }^{10}$. The sensitivity reached so far $^{11}$ in the search for the electric dipole moment of the neutron is $6.8 \times 10^{-22} \mathrm{eV}$, or one Bohr rotation every six days.

In this Letter, we demonstrate that energy eigenstates in the gravity potential of the earth can be probed using a new resonance-spectroscopy technique, using neutrons bounced off a horizontal mirror. This spectroscopy technique has in common the property that a quantum-system is coupled to an external resonator. Quantum mechanical transitions with a characteristic energy exchange between the coupling and the energy-levels are observed on resonance. A novelty of this work is the fact that the quantum mechanical transition is driven by an oscillating field that does not directly couple an electromagnetic charge or moment to an electromagnetic field. Instead, we observe energy transfer on resonance that is based on gravity-quantum states coupled to a modulator. We have named this technique gravity resonance spectroscopy, because the energy difference between these states has a one-to-one correspondence to the frequency of the modulator, in analogy to the nuclear magnetic resonance technique, where the energy splitting of a magnetic moment in an outer magnetic field is related to the frequency of a radio-frequency field. This is possible because of the feature of the quantum bouncing ball ${ }^{12,13}$ that the levels are not equidistant in energy. The linear gravity potential leads to measured ${ }^{14-16}$ discrete non-equidistant energy eigenstates $|n\rangle$. A combination of any two states can therefore be treated as a two-level system, as each transition can be addressed by its unique energy splitting or, in our case, by vibrating the mirror mechanically at the appropriate frequency. It has also been proposed to realize transitions between gravitational quantum states by means of oscillating magnetic gradient fields ${ }^{17}$. The physics behind these transitions is related to earlier studies of energy transfer where matter waves bounce off a vibrating mirror ${ }^{18,19}$ or a time-dependent crystal $^{20,21}$. In the latter case the transitions are between continuum states, in the quantum bouncer the transitions are between discrete eigenstates. Optical dipole traps of atoms are reviewed in ref. 22.

A measured energy level splitting $\Delta E$ can be compared with the prediction in the gravity potential, allowing a test of Newton's law at short distances. The experiments are linked to current ideas in string theories with large volume compactifications ${ }^{4,5}$ and/or cosmology $y^{23,24}$ and can rule out or substantiate forces that are possible via Abelian gauge fields in the bulk ${ }^{4,5}$, or via a pseudo-scalar coupling of axions, which are serious dark matter candidates in the previously experimentally inaccessible astrophysical axion window. Limits from our previous experiments with ultra-cold neutrons can be found elsewhere ${ }^{25-28}$. Owing to the nature of the Schrödinger equation, it also, in principle, provides a new test of the equivalence of inertial and gravitational mass ${ }^{3}$.

In general, the gravity-resonance-spectroscopy method consists of the three steps shown in Fig. 1a. In region one the initial state $|\mathrm{p}\rangle$ is prepared by a state selector. In region two a so-called $\pi$-pulse induces a transition into a second state $|\mathrm{q}\rangle$. In region three another state selector selects the state finally measured by the detector. In our experiments, all three regions are realized in a single device consisting of a polished mirror on the bottom and a rough scatterer on top (Figs $1 \mathrm{~b}$ and 2). The scatterer suppresses the population of higher levels by scattering and absorption. It allows only the ground state to pass and prepares the state $|\mathrm{p}\rangle$. Transitions to level $|\mathrm{q}\rangle$ are induced by vibrating the whole system in the vertical direction. The population of level $|\mathrm{q}\rangle$ is again destroyed by the state selector, leaving the remaining population of $|p\rangle$ to be detected. The lifetime-limiting interactions of the scatterer are described phenomenologically by adding decay terms to the equations of motion termed as damped oscillation (see Methods).

\footnotetext{
${ }^{1}$ Atominstitut, Technische Universität Wien, Stadionallee 2, 1020 Vienna, Austria, ${ }^{2}$ Institut Laue-Langevin, 6, Rue Jules Horowitz, 38042 Grenoble Cedex 9, France, ${ }^{3}$ E18, Physikdepartment, Technische Universität München, 85748 Garching, Germany, ${ }^{4}$ Physikalisches Institut, Universität Heidelberg, Philosophenweg 12, 69120 Heidelberg, Germany. *e-mail: abele@ati.ac.at.
} 
a

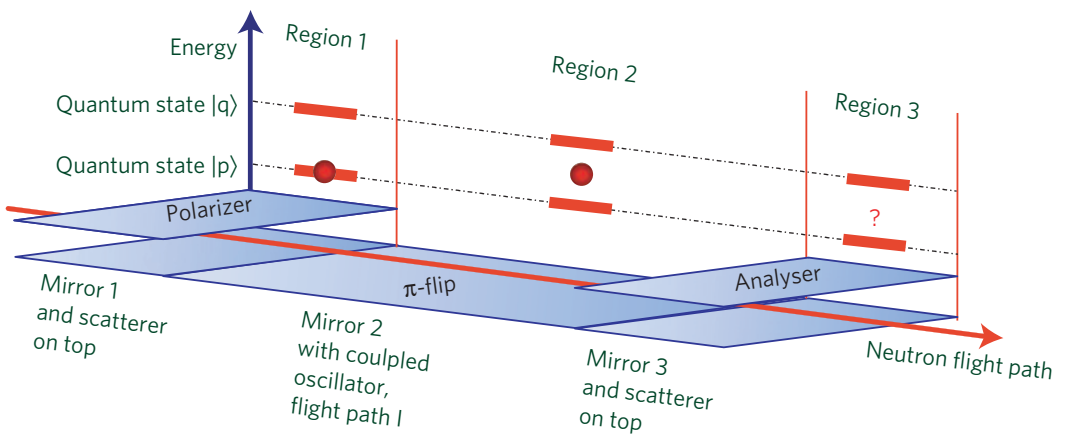

b

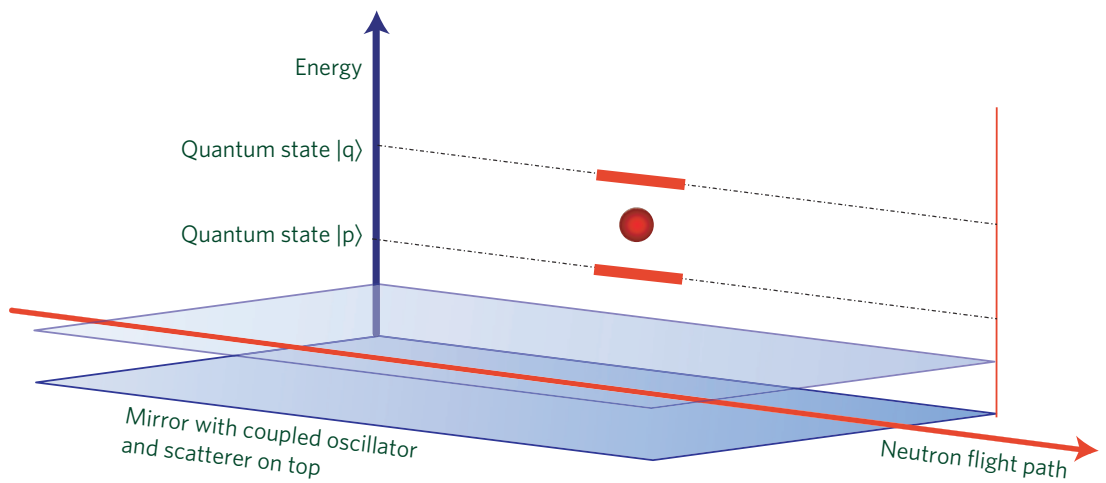

Figure 1 | Principle of gravity resonance spectroscopy and its experimental realization in our current set-up. a, Generally, one has to realize a state selector as polarizer, a so called $\pi$-flip, creating the superposition of the two states $|p\rangle$ and $|q\rangle$, of which the energy difference $\Delta E$ is to be measured, and a second state detector as an analyser. A polished mirror on the bottom and a rough absorbing mirror on top at a height of about $20 \mu \mathrm{m}$ is a realization of a state selector for the gravitational ground state of an ultra-cold neutron $|p\rangle$ (ref. 13). Higher, unwanted states are scattered out of the system and absorbed. The oscillator is installed in region 2. Here, transitions between quantum states $|p\rangle$ and $|q\rangle$ are induced within a time $t$ according to equation (2). In region 3, a second state selector, serving only as an analyser, transmits neutrons in state $|p\rangle$. A detector behind this system counts the transmitted neutrons. $\mathbf{b}$, In our current set-up, regions 1-3 are combined by using one neutron mirror on the bottom, one state-selector mirror on top, and a neutron detector behind the set-up. While neutrons are prepared in the ground state $|p\rangle$, the entire system is vibrating with frequency $v$ and strength $a$. If $E=h v$ matches the resonance condition given in equation (1), transitions between state $|p\rangle$ and $|q\rangle$ are induced. The state selector on top introduces an asymmetry, because state $|p\rangle$ passes the system with higher probability than state $|q\rangle$. A significant, vibration strength-dependent, drop in transmission therefore occurs at resonance.

The mirror is made of polished optical glass and is mounted on a piezo-driven vibration system. The experiment itself is mounted on a polished plane granite slab with active and passive anti-vibration tables underneath. The slab is levelled with a precision better than $1 \mu \mathrm{rad}$. A mu-metal shield suppresses the coupling of residual fluctuations of the magnetic field to the magnetic moment of the neutron adequately. As in previous measurements ${ }^{14-16}$, the neutrons are taken from the ultra-cold neutron installation PF2 at Institut Laue-Langevin (ILL). We restrict the horizontal velocity to $5.7 \mathrm{~m} \mathrm{~s}^{-1}<v<7 \mathrm{~m} \mathrm{~s}^{-1}$.

To test our state selector, we analysed the spatial neutron density distribution with a spatial resolution detector directly after the mirror and for a scatterer system without vibrations. The measurement agrees well with the quantum mechanical prediction $^{16}$ for our geometric parameters. $57 \%$ of the neutrons were found in the ground state, $37 \%$ in the second state, and $6 \%$ in higher states. To optimize the contrast in later measurements, we chose levels $|1\rangle$ and $|3\rangle$ as states $|\mathrm{p}\rangle$ and $|\mathrm{q}\rangle$ respectively.

Within the qBounce ${ }^{12,13}$ experiment, we performed two resonance-spectroscopy measurements with different geometric parameters (mirror length $L$ and gap height $h$ ), resulting in different resonance frequencies and widths, as outlined in Fig. 2. In general, the oscillator frequency at resonance for a transition between states with energies $E_{\mathrm{p}}$ and $E_{\mathrm{q}}$ is:

$$
\omega_{\mathrm{pq}}=\frac{E_{\mathrm{q}}-E_{\mathrm{p}}}{\hbar}=\omega_{\mathrm{q}}-\omega_{\mathrm{p}}
$$

Here, one has to take into account that because of the additional potential of the state selector, the energy eigenvalues depend on the gap height $h$, see Fig. 2 . Although the first energy level stays practically unchanged, higher states are more strongly affected.

The transition probability $|\mathrm{p}\rangle$ to $|\mathrm{q}\rangle$ is given in the Methods section. The transfer is referred to as Rabi transition. In our experiments we chose states $|1\rangle$ and $|3\rangle$ as a two-level system. In resonance, if the modulation frequency $\omega$ equals the transition frequency $\omega_{13}=\omega_{3}-\omega_{1}$, the system is driven into a coherent superposition of state $|1\rangle$ and $|3\rangle$ and we can choose amplitude $a$ in such a way that we have complete reversal of the state occupation.

The observable is the measured transmission left after the transition $|1\rangle$ to $|3\rangle$ as a function of the modulation frequency $\omega$ and amplitude $a$, see Fig. 3. During the time the neutron spends on the mirror, the acceleration on the mirror is practically constant and does not change significantly. We measure $a$ with a noise and vibration analyser attached to the neutron mirror system. In addition, the position-dependent mirror vibrations were measured using a laser-based vibration analysis system. The piezo-system by itself does not show significant self-resonant behaviour, which might influence the neutron transmission in the frequency range considered.

For the first experiment, Fig. 3a shows the measured count rate as a function of $\omega$. Blue (brown) data points correspond to measurements with moderate (high) vibration strength $1.5 \leq a \leq$ $4.0 \mathrm{~m} \mathrm{~s}^{-2}\left(4.9 \leq a \leq 7.7 \mathrm{~m} \mathrm{~s}^{-2}\right)$. The corresponding Rabi resonance curve was calculated using their mean vibration strength of 

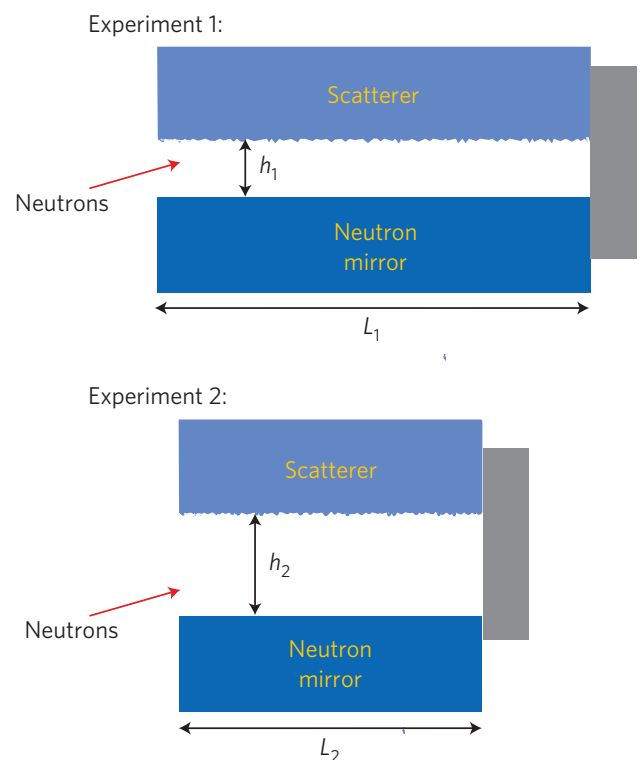

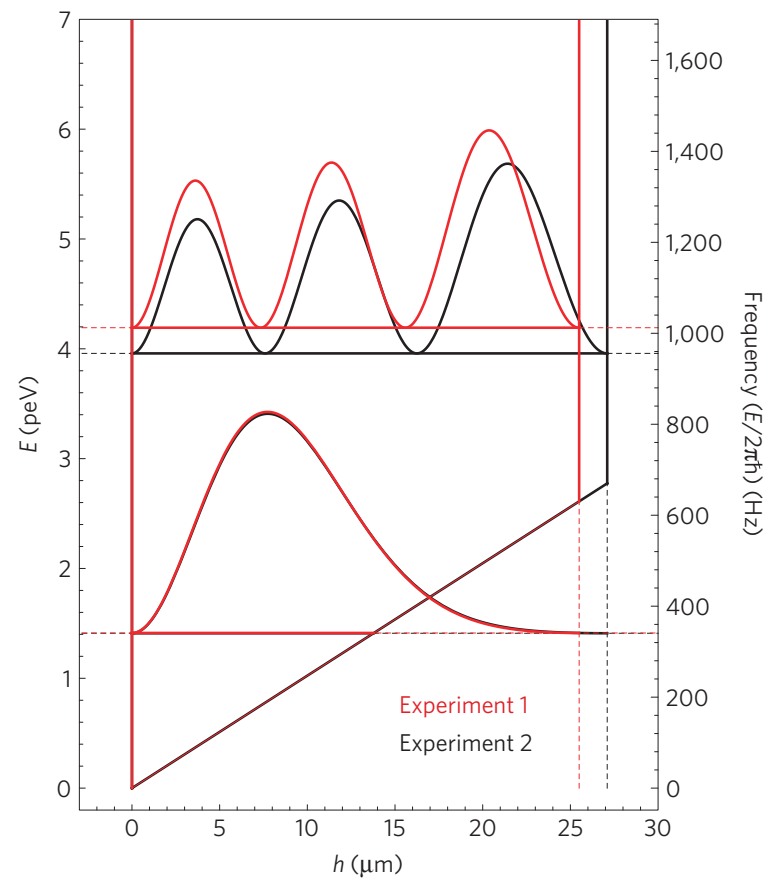

Set-up parameters and experimental results
\begin{tabular}{|l|l|l|l|l|l|l|l|}
\hline & $\begin{array}{l}\text { Length of the } \\
\text { neutron mirror }\end{array}$ & $\begin{array}{l}\text { Height } \\
\text { of } \\
\text { scatterer } \\
\text { Length } L(\mathrm{~cm})_{x} \\
\text { Width } W(\mathrm{~cm})_{x} \\
\text { Height } H(\mathrm{~cm})\end{array}$ & $\begin{array}{l}\text { Mean } \\
\text { time of } \\
\text { flight }\end{array}$ & $\begin{array}{l}\text { Energy } \\
\text { difference }\end{array}$ & $\begin{array}{l}\text { Resonance } \\
\text { frequency } \\
\text { (prediction) }\end{array}$ & $\begin{array}{l}\text { Resonance } \\
\text { frequency } \\
\text { (measurement) }\end{array}$ & $\begin{array}{l}\text { Resonance width } \\
\text { (FWHM) }\end{array}$ \\
\hline Experiment 1 & $15 \times 3 \times 3$ & 25.5 & 23 & 2.78 & $E_{13}(\mathrm{peV})$ & $\omega_{13}(\mathrm{~Hz})$ & $\Delta \omega(\mathrm{Hz})$ \\
\hline Experiment 2 & $10 \times 3 \times 3$ & 27.1 & 15 & 2.55 & $2 \pi \times 671$ & $2 \pi \times(705 \pm 6)$ & $2 \pi \times 41.2$ \\
\hline
\end{tabular}

Figure 2 | Quantum states of an ultra-cold neutron bound in the gravity potential of the earth and a reflecting mirror. Energy eigenvalues (horizontal lines) and neutron density distributions are displayed for level $|1\rangle$ and $|3\rangle$. A state selector is placed on top, above the mirror, removing higher, unwanted states. The state selector is placed at height $h_{1}=25.5 \mu \mathrm{m}$ (red) in the first measurement, and at height $h_{2}=27.1 \mu \mathrm{m}$ (black) in the second measurement. The position of the state selector defines a second boundary condition for the neutron density distributions. It allows us to tune the resonance frequency by

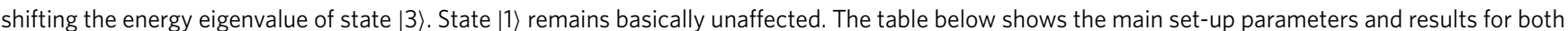
versions of the experiment.

$2.95 \mathrm{~m} \mathrm{~s}^{-2}\left(5.87 \mathrm{~m} \mathrm{~s}^{-2}\right)$. The black data point sums up all of the measurements at zero vibration. The grey band represents the one sigma uncertainty of all off-resonant data points. The brown line is the quantum expectation as a function of oscillator frequency $\omega$ for Rabi transitions between state $|1\rangle$ and state $|3\rangle$ within an average time of flight $\tau=L / v=23 \mathrm{~m} \mathrm{~s}$. The normalization for transmission $T$, frequency at resonance $\omega_{13}$ and global parameter $f$ are the only fitting parameters. $f$ is a multiplicator for the vibration amplitude and takes into account that the amplitude is not uniform over the whole surface of the mirror. The averaged effective amplitude acting on the neutron is somewhat smaller than the measured one and was found to be $f=0.56 \pm 0.16$. It is a global parameter, independent of the vibration frequency, and can therefore easily be corrected for. A sharp resonance was found at frequency $\omega_{13}=2 \pi \times(705 \pm 6) \mathrm{Hz}$, which is close to the frequency prediction of $\omega_{13}=2 \pi \times 671 \mathrm{~Hz}$, remembering that the gap height measurement has an uncertainty due to the roughness of the state selector. The full-width at halfmaximum is the prediction made on the basis of the time the neutrons spend in the modulator. The prediction was convoluted by the time of flight distribution function. The significance for $|1\rangle \rightarrow|3\rangle$ excitations is 3.5 standard deviations.
In the second measurement, the reduced length reduces the average flight time to $\tau=15 \mathrm{~m} \mathrm{~s}$. The gap height $h$ is $1.6 \mu \mathrm{m}$ larger, thus changing the predicted resonant frequency to $\omega_{13}=615 \mathrm{~Hz}$. We observe a resonance frequency $\omega_{13}=2 \pi \times(592 \pm 11) \mathrm{Hz}$, close to the prediction, and $f=0.99 \pm 0.29$. Figure $3 \mathrm{~b}$ shows the combined result for measurements with both mirror length $L=10 \mathrm{~cm}$ and $L=15 \mathrm{~cm}$. The transmission in units of the unperturbed system is displayed as a function of detuning.

In total, the significance for gravity resonance spectroscopy between state $|1\rangle$ and $|3\rangle$ at $\omega_{13}$ is 4.9 standard deviations. The left and right hand data points combine all off-resonant measurements with $\left|\left(\omega-\omega_{13}\right) / \Delta \omega\right| \geq 1.5$, where $\Delta \omega$ is the full-width at half-maximum. The statistical sensitivity of the measured energy difference between the gravity level $|1\rangle$ and $|3\rangle$ is $7.6 \times 10^{-3}$, corresponding to $\Delta E=2 \times 10^{-14} \mathrm{eV}$. The limiting systematic uncertainty in this experiment is the roughness of the state selector $(0.4 \mu \mathrm{m})$.

The new method profits from small systematic effects in such systems, mainly owing to the fact that, in contrast to atoms, the electrical polarizability of neutrons is extremely low. Neutrons are not disturbed by short range electric forces such as van der Waals 

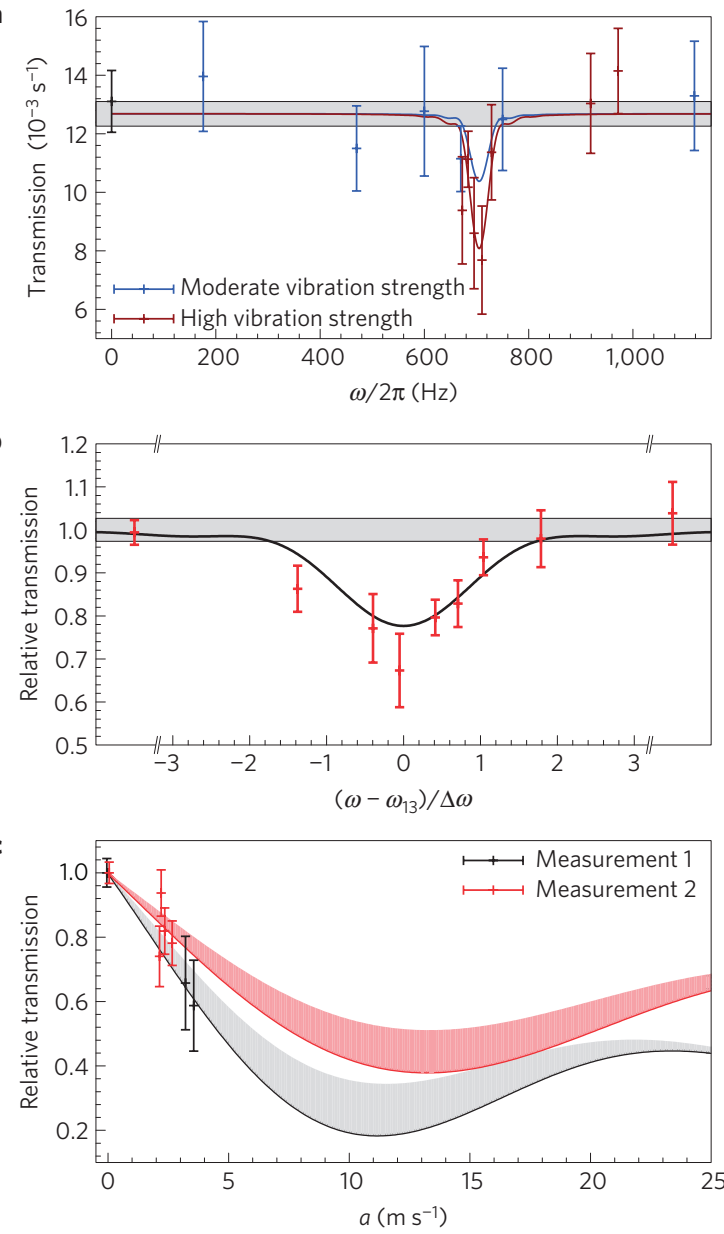

Figure 3 | Gravity resonance spectroscopy and excitation-experimental results. a, The transmission as a function of modulation frequency shows a sharp resonance at $\omega_{13}=2 \pi \times(705 \pm 6) \mathrm{s}^{-1}$. The grey band represents the statistical $1 \sigma$ uncertainty of all off-resonant data points. Blue (brown) data points correspond to measurements with moderate (high) vibration strength $1.5 \leq a \leq 4.0 \mathrm{~m} \mathrm{~s}^{-2}\left(4.9 \leq a \leq 7.7 \mathrm{~m} \mathrm{~s}^{-2}\right)$. The corresponding Rabi resonance curve is calculated using the corresponding mean vibration strength of $2.95 \mathrm{~m} \mathrm{~s}^{-2}\left(5.87 \mathrm{~m} \mathrm{~s}^{-2}\right)$. The black data point sums up all of the measurements at zero vibration. $\mathbf{b}$, Combined result for Measurements 1 and 2. The transmission in units of the unperturbed system is displayed as a function of detuning normalized to the anticipated resonance width. The significance for the observation of gravity spectroscopy between state |1 and $|3\rangle$ at $\omega_{13}$ is 4.9 standard deviations. The left and right data points combine all off-resonant measurements with $\left|\left(\omega-\omega_{13}\right) / \Delta \omega\right| \geq 1.5$. c, Measured damped Rabi oscillation as a function of $\Omega_{R}$ converted in vibration strength in units of $\left(\mathrm{m} \mathrm{s}^{-2}\right)$ and extrapolation to higher vibration strengths according to equation (3). In resonance, the neutron transmission decreases as expected in the same frequency band $\left|\left(\omega-\omega_{13}\right) / \Delta \omega\right| \leq 0.3$. This effect is visible for both experiments (black and red). The shorter mirror length in the second measurement results in a reduced sensitivity to $|1\rangle \leftrightarrow|3\rangle$ transitions, and the Rabi oscillation reaches its minimum at higher vibration amplitudes. All error bars shown in this figure represent the statistical $1 \sigma$ standard deviation.

or Casimir forces. Together with its neutrality, this provides the key to a sensitivity of several orders of magnitude below the strength of electromagnetism ${ }^{29}$. The long term plan is to apply Ramsey's method of separated oscillating fields ${ }^{30}$ with an expansion of the current set-up, which works without any influence of the state selector on the energy analysis.

\section{Methods}

Rabi resonance spectroscopy ${ }^{1}$ measures the energy difference between a two-level system $|\mathrm{p}\rangle$ and $|\mathrm{q}\rangle$ with a coupled oscillating field $\omega$ and damping $\gamma$. The wave function of the two level system is:

$$
\psi(\bar{r}, t)=\langle\bar{r} \mid \psi(t)\rangle=C_{\mathrm{p}}(t) \exp \left(-i \omega_{\mathrm{p}} t\right) u_{\mathrm{p}}(\bar{r})+C_{\mathrm{q}}(t) \exp \left(-i \omega_{\mathrm{q}} t\right) u_{\mathrm{q}}(\bar{r})
$$

with the time-varying coefficients $C_{\mathrm{p}}(t)$ and $C_{\mathrm{q}}(t)$.

With the frequency difference $\omega_{\mathrm{pq}}$ between the two states, the frequency $\omega$ of the driving field, the detuning $\delta \omega=\omega_{\mathrm{pq}}-\omega$, the Rabi frequency $\Omega_{\mathrm{R}}$ and the time $t$, the coupling between the time-varying coefficients is given by:

$$
\frac{\mathrm{d}}{\mathrm{d} t}\left(\begin{array}{c}
\tilde{C}_{\mathrm{p}}(t) \\
\tilde{C}_{\mathrm{q}}(t)
\end{array}\right)=\frac{i}{2}\left(\begin{array}{cc}
\delta \omega & \Omega_{\mathrm{R}} \\
\Omega_{\mathrm{R}}^{*} & -\delta \omega
\end{array}\right)\left(\begin{array}{c}
\tilde{C}_{\mathrm{p}}(t) \\
\tilde{C}_{\mathrm{q}}(t)
\end{array}\right)
$$

with a transformation into the rotating frame of reference:

$$
\begin{aligned}
& C_{\mathrm{p}}(t)=\tilde{C}_{\mathrm{p}}(t) \cdot \mathrm{e}^{-i \delta \omega t / 2} \\
& C_{\mathrm{q}}(t)=\tilde{C}_{\mathrm{q}}(t) \cdot \mathrm{e}^{i \delta \omega t / 2}
\end{aligned}
$$

$\Omega_{\mathrm{R}}$ is a measure of the strength of the coupling between the two levels and is related to the vibration strength $a$ via $^{30}$ :

$$
\Omega_{\mathrm{R}}=i \frac{a}{\omega}\left\langle\mathrm{p}\left|\frac{\partial}{\partial z}\right| \mathrm{q}\right\rangle
$$

The probability of being found in the excited state as a function of time is:

$$
P(t)=\left(\frac{\Omega_{\mathrm{R}}}{\Omega_{\mathrm{R}}^{\prime}}\right)^{2} \sin ^{2}\left(\frac{\Omega_{\mathrm{R}}^{\prime}}{2} t\right)
$$

where the effective Rabi frequency is:

$$
\Omega_{\mathrm{R}^{\prime}}=\sqrt{\Omega_{\mathrm{R}}^{2}+\delta \omega^{2}}
$$

Such oscillations are damped out and the damping rate depends on how strongly the system is coupled to the environment. In our case, the damping is caused by the state selector at height $h$ above a mirror. A key point for the demonstration of this method is that it allows the detection of resonant transitions $|\mathrm{p}\rangle \leftrightarrow|\mathrm{q}\rangle$ at a frequency that is tuned by the height of the state selector. The additional mirror potential shifts the energy of state $|3\rangle$ as a function of height, see Fig. 2. The absorption is described phenomenologically by adding decay terms $\gamma_{p}$ and $\gamma_{\mathrm{q}}$ to the equations of motion:

$$
\frac{\mathrm{d}}{\mathrm{d} t}\left(\begin{array}{l}
\tilde{C}_{\mathrm{p}}(t) \\
\tilde{C}_{\mathrm{q}}(t)
\end{array}\right)=\frac{i}{2}\left(\begin{array}{ll}
\left(\delta \omega+i \gamma_{\mathrm{p}}\right) & \Omega_{\mathrm{R}} \\
\Omega_{\mathrm{R}}^{*} & \left(-\delta \omega+i \gamma_{\mathrm{q}}\right)
\end{array}\right)\left(\begin{array}{l}
\tilde{C}_{\mathrm{p}}(t) \\
\tilde{C}_{\mathrm{q}}(t)
\end{array}\right)
$$

The solution of this equation is given by:

$$
\begin{gathered}
\left(\begin{array}{c}
\tilde{C}_{\mathrm{p}}(t) \\
\tilde{C}_{\mathrm{q}}(t)
\end{array}\right)=\left(\begin{array}{cc}
\cos \left(\frac{\Omega_{\mathrm{R}}^{\prime} t}{2}\right)+\left(\frac{\frac{1}{2}\left(\gamma_{\mathrm{q}}-\gamma_{\mathrm{p}}\right)+i \delta \omega}{\Omega_{\mathrm{R}}^{\prime}}\right) \sin \left(\frac{\Omega_{\mathrm{R}}^{\prime} t}{2}\right) & i\left(\frac{\Omega_{\mathrm{R}}}{\Omega_{\mathrm{R}}^{\prime}}\right) \sin \left(\frac{\Omega_{\mathrm{R}}^{\prime} t}{2}\right) \\
i\left(\frac{\Omega_{\mathrm{R}}^{*}}{\Omega_{\mathrm{R}}^{\prime}}\right) \sin \left(\frac{\Omega_{\mathrm{R}}^{\prime} t}{2}\right) & \cos \left(\frac{\Omega_{\mathrm{R}}^{\prime} t}{2}\right)-\left(\frac{\frac{1}{2}\left(\gamma_{\mathrm{q}}-\gamma_{\mathrm{p}}\right)+i \delta \omega}{\Omega_{\mathrm{R}}^{\prime}}\right) \sin \left(\frac{\Omega_{\mathrm{R}}^{\prime} t}{2}\right)
\end{array}\right) \\
\cdot \mathrm{e}^{-1 / 4\left(\gamma_{\mathrm{p}}+\gamma_{\mathrm{q}}\right) t} \cdot\left(\begin{array}{c}
\tilde{C}_{\mathrm{p}}(0) \\
\tilde{C}_{\mathrm{q}}(0)
\end{array}\right)
\end{gathered}
$$

The fit used in Fig. 3a contains three parameters, the resonant frequency $\omega_{\mathrm{pq}}$, the transmission normalization $N$, and vibration strength parameter $f$, which is multiplied by the measured acceleration. The damping as a function of $\Omega_{\mathrm{R}}$ was measured separately, see Fig. $3 c$, as well as the width of the Rabi oscillation and background $\left(0.0050 \pm 0.0002 \mathrm{~s}^{-1}\right)$.

Received 23 August 2010; accepted 9 March 2011; published online 17 April 2011

\section{References}

1. Rabi, I. I., Millman, S., Kusch, P. \& Zacharias, J. R. The molecular beam resonance method for measuring nuclear magnetic moments. The magnetic moments of ${ }_{3} \mathrm{Li}^{6}{ }_{3} \mathrm{Li}^{7}$ and ${ }_{9} \mathrm{~F}^{19}$. Phys. Rev. 55, 526-535 (1939).

2. Ramsey, N. F. Molecular Beams 1985 edn (paperback edition) 124-134 (Clarendon Press, 1956).

3. Kajari, E., Harshman, N. L., Rasel, E. M., Stenholm, S., Süßmann, G. \& Schleich, W. P. Inertial and gravitational mass in quantum mechanics. Appl. Phys. B 100, 43-60 (2010).

4. Arkani-Hamed, N., Dimopolos, S. \& Dvali, G. Phenomenology, astrophysics and cosmology of theories with submillimetre dimensions and $\mathrm{TeV}$ scale quantum gravity. Phys. Rev. D 59, 086004 (1999).

5. Antoniadis, I. Physics with large extra dimensions and non-newtonian gravity at sub-mm distances. Lect. Notes Phys. 631, 337-354 (2003). 
6. Ramsey, N. F. Experiments with separated oscillatory fields and hydrogen masers. Rev. Mod. Phys. 62, 541-552 (1990).

7. Cronin, A., Schmiedmayer, J. \& Pritchard, D. Optics and interferometry with atoms and molecules. Rev. Mod. Phys. 81, 1051-1129 (2009).

8. Vandersypen, L. M. K. \& Chuang, I. L. NMR techniques for quantum control and computation. Rev. Mod. Phys. 76, 1037-1069 (2005).

9. Roos, C. F., Chwalla, M., Kim, K., Riebe, M. \& Blatt, R. Designer atoms' for quantum metrology. Nature 443, 316-319 (2006).

10. De Kieviet, M., Dubbers, D., Schmidt, C., Scholz, D. \& Spinola, U. ${ }^{3}$ He Spin Echo: New atomic beam technique for probing phenomena in the neV range. Phys. Rev. Lett 75, 1919-1922 (1995).

11. Baker, C. A. et al. An improved experimental limit on the electric dipole moment of the neutron. Phys. Rev. Lett 97, 131801 (2006).

12. Abele, H., Jenke, T., Stadler, D. \& Geltenbort, P. QuBounce: The dynamics of ultra-cold neutrons falling in the gravity potential of the Earth. Nucl. Phys. A827, 593c-595c (2009).

13. Jenke, T., Stadler, D., Abele, H. \& Geltenbort, P. Q-BOUNCE-experiments with quantum bouncing ultracold neutrons. Nucl. Instr. Meth. A 611, 318-323 (2009).

14. Nesvizhevsky, V. V. et al. Quantum states of neutrons in the Earth's gravitational field. Nature 415, 297-299 (2002).

15. Nesvizhevsky, V. V. et al. Study of neutron quantum states in the gravity field. Eur. Phys. J. C40, 479-491 (2005).

16. Westphal, A. et al. A quantum mechanical description of the experiment on the observation of gravitationally bound states. Eur. Phys. J. C51, 367-375 (2007).

17. Kreuz, M. et al. A method to measure the resonance transitions between the gravitationally bound quantum states of neutrons in the GRANIT spectrometer. Nucl. Instr. Meth. Phys. Res. A 611, 326-330 (2009).

18. Hamilton, W. A., Klein, A. G., Opat, G. I. \& Timmins, P. A. Neutron diffraction by surface acoustic waves. Phys. Rev. Lett. 58, 2770-2773 (1987).

19. Felber, J., Gähler, R., Rausch, C. \& Golub, R. Matter waves at a vibrating surface: Transition from quantum-mechanical to classical behaviour. Phys. Rev. A53, 319-328 (1996).

20. Steane, A., Szriftgiser, P., Desbiolles, P. \& Dalibard, J. Phase modulation of atomic de Broglie waves. Phys. Rev. Lett. 74, 4972-4975 (1995).

21. Bernet, S., Oberthaler, M. K., Abfalterer, R., Schmiedmayer, J. \& Zeilinger, A. Coherent frequency shift of atomic matter waves. Phys. Rev. Lett. 77, 5160-5163 (1996)
22. Grimm, R., Weidemüller, M. \& Ovchinnikov, Y. B. Optical dipole traps for neutral atoms. Adv. Atomic Mol. Opt. Phys. 42, 95-170 (2000).

23. Callin, P. \& Burgess, C. P. Deviations from Newton's law in supersymmetric large extra dimensions. Nucl. Phys. B752, 60-79 (2006).

24. Sundrum, R. Towards an effective particle-string resolution of the cosmological constant problem. J. High Energy Phys. 07, 001 (1999).

25. Westphal, A., Abele, H. \& Baeßler, S. Analytically derived limits on short-range fifth forces from quantum states of neutrons in the Earth's gravitational field. Preprint at http://arxiv.org/abs/hep-ph/0703108.

26. Baeßler, S., Nesvizhevsky, V. V., Protasov, K. V. \& Voronin, A. Y. Constrain on the coupling of axionlike particles to matter via an ultracold neutron gravitational experiment. Phys. Rev. D 75, 075006 (2007).

27. Abele, H., Baessler, S. \& Westphal, A. in Quantum Gravity-from Theory to Experimental Search' (ed. Lämmerzahl, C.) (Lect. Notes Phys., Vol. 631, Springer, 2003).

28. Nesvizhevsky, V. V. \& Protasov, K. V. Constraints on non-Newtonian gravity from the experiment on neutron quantum states in the earth's gravitational field. Classical Quant. Gravity 21, 4557-4566 (2004).

29. Abele, H. The neutron. Its properties and basic interactions. Prog. Part. Nucl. Phys. 60, 1-81 (2008).

30. Abele, H., Jenke, T., Leeb, H. \& Schmiedmayer, J. Ramsey's method of separated oscillating fields and its application to gravitationally induced quantum phaseshifts. Phys. Rev. D 81, 065019 (2010).

\section{Acknowledgements}

We gratefully acknowledge support from the Austrian Science Fund (FWF) under Contract No. I529-N20 and the German Research Foundation (DFG) as part of the Priority Programme (SPP) 1491 'Precision experiments in particle and astrophysics with cold and ultracold neutrons', the DFG Excellence Initiative 'Origin of the Universe', and DFG support under Contract No. Ab128/2-1. The neutron mirrors were characterized by S-DH Sputterdünnschichttechnik, Heidelberg.

\section{Author contributions}

All of the authors made a substantial contribution to this work.

\section{Additional information}

The authors declare no competing financial interests. Supplementary information accompanies this paper on www.nature.com/naturephysics. Reprints and permissions information is available online at http://npg.nature.com/reprintsandpermissions. Correspondence and requests for materials should be addressed to H.A. 